\title{
Piezoelectric Cantilevers for Energy Harvesting with Irregular Design of the Cross Sections
}

\author{
Andrius ČEPONIS*, Dalius MAŽEIKA**, Genadijus KULVIETIS***, Ying YANG**** \\ *Department of Electrical Engineering, Vilnius Collage of Technologies and Design, Olandu st. 16, Vilnius, Lithuania, \\ LT-01100,E-mail: a.ceponis@vtdko.lt \\ **Department of Information Systems, Vilnius Gediminas Technical University, Sauletekio avn. 11, Vilnius, Lithuania, \\ LT-10223, E-mail: dalius.mazeika@vgtu.lt \\ *** Department of Information Technologies, Vilnius Gediminas Technical University, Saulètekio avn. 11, Vilnius, \\ Lithuania, LT-10223, E-mail: genadijus.kulvietis@vgtu.lt \\ ****State Key Laboratory of Mechanics and Control of Mechanical Structures, Nanjing University of Aeronautics and As- \\ tronautics, PO Box 359, 29 Yudao Street, Nanjing, Jiangsu 210016, China, E-mail: yingyang@nuaa.edu.cn
}

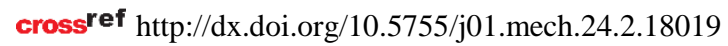

\section{Introduction}

Kinetic energy harvesting devices allows to reuse wasted mechanical energy and transform it to electrical energy. Every moving object has kinetic energy and as for example, vibrations of refrigerator, personal computer, milling machine and etc., can be harvested using energy harvesting technologies [1]. Kinetic energy harvesters ensure opportunity to develop lifetime power supply for numerous low power electronic devices [2].

Kinetic energy can be harvested by electromagnetic, electrostatic, triboelectric and piezoelectric transducers. Electromagnetic, electrostatic and triboelectric transducers have low power density and complex constructions. Moreover, operation principle of the electrostatic transducers is based on using external power source [3, 4]. These disadvantages of the electromagnetic, electrostatic and triboelectric transducers lead to complicated their implementation for practical applications. On the other hand, kinetic energy harvesters based on piezoelectric materials are more promising due to high power density, simple construction, low cost and etc. [5].

Design of piezoelectric energy harvesting devices usually are based on multilayer cantilevers with one or two piezoceramic layers. Efficiency of such system depends on strain distribution created at the piezo ceramic layers [6]. A lot of reports are made on investigation of different design of cantilevers with the goal to increase strain value and to make strain distribution more even in the piezo ceramic layer. Muthalif and Nordin [7] announced the experimental and analytical investigations of the piezoelectric cantilevers with different shapes. Authors analysed efficiency of the proposed designs. Investigation was performed using rectangular, trapezoidal and triangle shape piezoelectric cantilevers. Analysis of the obtained results revealed that the best efficiency was achieved using piezoelectric cantilever with triangle shape. On the other hand, authors did not analysed possibility to increase efficiency of the energy harvesters by modifying cross section design of the piezoelectric cantilevers. Paquin and St-Amant investigated electromechanical characteristics of the piezoelectric cantilevers with variable thickness [8]. Investigation was based on Rayleigh-Ritz approximations with a trigonometric function. Finite element modelling (FEM) revealed that tapered cantilever has more uniform strain distribution along piezoelectric layer. Authors reported that proposed modification of cross section allowed to increase efficiency of energy harvester by factor 3.6. Reddy et al. proposed possibility to improve strain and it distribution in the piezoelectric cantilever by trapezoidal cavity which was placed under piezo ceramic layer [9]. Authors claimed that proposed design of the cross section area provides higher output voltage and allows increasing efficiency of harvester. Analytical and experimental investigation revealed that the output voltage was increased more than $100 \%$ compare to the conventional piezoelectric cantilever. Guan et al. proposed design of $\mathrm{H}$ type seismic mass. An additional rotation moment created by $\mathrm{H}$ type seismic mass leads to more uniform strain distribution at the piezoelectric cantilever [10]. Numerical and experimental investigation showed that proposed design of the $\mathrm{H}$ type seismic mass allows increasing strain of piezoelectric layer and efficiency of harvester as well. Xu et al. investigated piezoelectric cantilever with a right angle structure [11]. The special design of the structure creates a large rotation moment at the tip of piezoelectric cantilever. Authors concluded that proposed solution dramatically improves strain distribution at the piezoelectric cantilever. Based on results of numerical and experimental investigation authors claimed that relative utilization efficiency of piezo ceramic layer was improved more than $43 \%$ compare to the conventional piezoelectric cantilever. Ma and et al. proposed special design of the seismic mass, based on compliant mechanism. Authors concluded that such design increases strain of the cantilever [12]. The main goal of the proposed design was to improve efficiency of the piezoelectric cantilever by obtaining quadratic boundary conditions. Such boundary conditions ensure that the first vibration mode has the shape that is close to parabola. Authors performed modelling and experimental investigation which revealed that proposed design ensures 2 times higher output voltage and 4 times higher output power compare to the conventional piezoelectric cantilevers.

This paper represents numerical and experimental investigations of the rectangular cantilevers with irregular design of the cross section area. Three different designs of the cross section area are proposed. Modified profiles of the cross section area reduce stiffness of the cantilevers and ensures gain of strain and strain distribution across the piezo ceramic layer. Therefore, modifications of cross section area 
improve electrical characteristics of the rectangular cantilevers.

\section{Design of piezoelectric cantilevers with irregular cross section}

In most cases piezoelectric cantilevers employed for energy harvesting vibrates at the first out of plane bending mode. Conventional piezoelectric cantilevers with rectangular cross section area has linear strain distribution along the length of cantilever and the largest strain value is obtained near the fixed end of cantilever. Moreover, strain and it distribution along piezo ceramic layer has influence to the electrical output characteristics of the piezoelectric cantilevers. Strain function of piezoelectric cantilever has the following expression [2]:

$$
\varepsilon(x) \frac{m(\ddot{y}+\ddot{z}) h}{2 Y_{\text {Piezo }} I(x)}\left(2 l_{b}-l_{e}\right),
$$

here $\varepsilon$ is strain; $m(\ddot{y}+\ddot{z})$ is vertical force acting at the free end of piezoelectric cantilever; $h$ is a distance between centres of cantilever and piezo electric layer; $Y_{\text {Piezo }}$ is Young modulus of the piezoelectric material; $l_{b}$ is length of the piezoelectric cantilever, $l_{e}$ is length of piezo ceramic layer covered by electrode; $I(x)$ is second moment of inertia as a function of distance $x$ from fixed end of piezoelectric cantilever. Function of second moment of inertia can be expressed as follows [2]:

$$
I(x)=2\left[\frac{w t_{c}^{3}}{12}+w t_{c} h^{2}(x)\right]+\frac{\eta_{s} w t_{s}^{3}(x)}{12},
$$

here $w$ is width of cantilever; $t_{c}$ is thickness of piezo ceramic layer; $h$ is distance between centres of cantilever and piezo ceramic layer as a function of distance $x$ from the fixed end of piezoelectric cantilever; $t_{s}$ is thickness of cantilever as a function of distance $x$ from the fixed end of piezoelectric cantilever; $\eta_{s}$ is ratio between of piezo ceramics and cantilevers materials Young`s modules and it can be expressed as $\eta_{s}=Y_{\text {Piezo }} / Y_{\text {Cantilevers. }}$ Relation between output voltage and strain can be written as follows [2]:

$$
U=\frac{d_{31} Y_{\text {Piezo }} b}{C_{p}} \int_{0}^{l} \varepsilon(x) d x,
$$

here $Y_{\text {Piezo }}$ is Young`s modulus of the piezo ceramic; $C_{p}$ is capacitance of piezo ceramic layer; $b$ is thickness of piezo ceramic layer; $d_{31}$ is piezoelectric coefficient; $\varepsilon(x)$ is strain function at piezo ceramic layer; $l$ is length of piezo ceramic layer. Analysing Eq. (3) it can be seen that output voltage value depends on the integral of strain function. On the other hand, strain function of piezoelectric cantilever is indirect proportional to a second moment of inertia function. Based on Eq. (2), function of second moment of inertia is related to the thickness of cantilever. Therefore, reduction of the thickness will cause a lower value of second moment of inertia and larger strain values of piezoelectric cantilever. Finally, analysis of the interaction between strain and output voltage showed that value of output voltage depends on second moment of inertia and cantilever thickness function therefore it was decided to make modifications in the cantilever cross-section design.

Periodical gaps with the cylindrical, rectangular and trapezoidal shape were introduced for this purpose. Such shapes of the gaps were chosen with the aim to reduce value of the second moment of inertia. Height of the gaps were set to $1 / 2$ of the cantilever thickness $t_{s}$. The gaps were placed under piezo ceramic layers. The periodicity of the gaps was chosen for each case individually so that the second moment of inertia will be minimized and crack possibility in the piezo ceramic layer will be avoided. Moreover, the conventional cantilever was designed for the reference and comparison. The schemes of the cross section designs and geometrical parameters of the cantilevers are given in Table 1 and Fig. 1, respectively.

Table 1

Geometrical parameters of the cantilevers

\begin{tabular}{|c|c|l|}
\hline Parameter & $\begin{array}{c}\text { Value } \\
(\mathrm{mm})\end{array}$ & \multicolumn{1}{|c|}{ Description } \\
\hline$w$ & 5 & Widths of the cantilevers \\
\hline$t_{s}$ & 1 & Thickness of the cantilevers \\
\hline$t_{c}$ & 0.5 & $\begin{array}{l}\text { Thickness of the piezo ceramic } \\
\text { layer }\end{array}$ \\
\hline$l_{e}$ & 30 & Length of the piezo ceramic layer \\
\hline$l_{b}$ & 50 & Length of the cantilever \\
\hline$l_{m}$ & 10 & Length of the seismic mass \\
\hline$l_{\text {cir }}$ & 29 & $\begin{array}{l}\text { Length of modified cross section } \\
\text { area }\end{array}$ \\
\hline$l_{\text {rec }}$ & 28.5 & $\begin{array}{l}\text { Length of modified cross section } \\
\text { area }\end{array}$ \\
\hline$l_{h e x}$ & 29.5 & $\begin{array}{l}\text { Length of modified cross section } \\
\text { area }\end{array}$ \\
\hline
\end{tabular}

It must be highlighted that the main geometrical parameters of all cantilevers are the same i.e. length, width and thickness. However, design of the cross sections is different (Fig. 1). Also, uniform seismic mass was used for all cantilevers. Weight of the mass was equal to $8.71 \mathrm{~g}$.

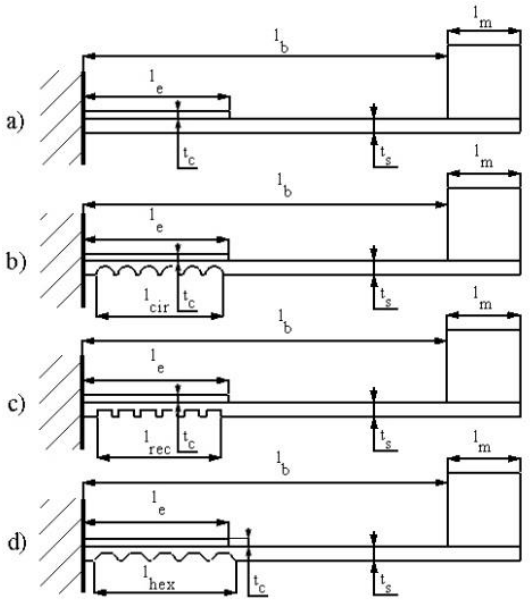

Fig. 1 Schematics of the cantilevers with irregular cross sections areas; a - conventional design of the cross section area; $b$ - cross section area modified by cylindrical gaps; $c$ - cross section area modified by rectangular gaps; $\mathrm{d}$ - cross section area modified by trapezoidal gaps 
Principle scheme of the piezoelectric energy harvesting system based on proposed cantilevers is shown in Fig. 2. The energy harvesting system consists of rectangular cantilever with piezo ceramic layer which was glued on the top surface of the cantilever. The seismic mass was attached to the tip of cantilever by bolt. (Figs. $2-6$ ). The plastic clamping frame with three bolts was used to clamp the cantilever at the one end and to fix whole system to the host.

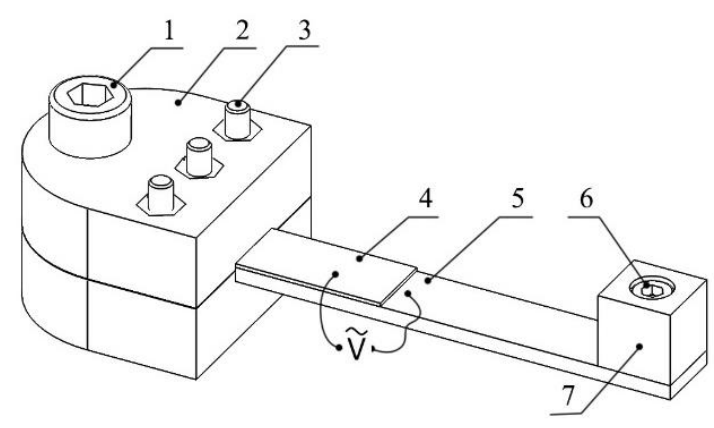

Fig. 2 Principle scheme of piezoelectric energy harvesting system; 1 - clamping bolt of the whole structure; $2-$ plastic support frame; 3 -clamping bolts for cantilever; 4 - piezo ceramic layer; 5 - cantilever; 6 clamping bolt for the seismic mass; 7 - seismic mass

\section{Numerical investigation of rectangular piezoelectric cantilevers with irregular cross section area}

Numerical investigation of the cantilevers with irregular cross section areas was performed. The aim of investigation was to analyse and assess mechanical and electrical characteristics of the proposed piezoelectric cantilevers and to compare results to the conventional case. Modal analyse of each cantilever was performed. Natural frequencies and modal shapes were obtained. Frequency response analysis was performed in order to obtain mechanical and electrical characteristics of the piezoelectric cantilevers. Finite element models (FEM) were built by Comsol Multiphysics 5.2 software. Properties of the materials and boundary conditions used for the models are given in Table 4 and Fig. 3.

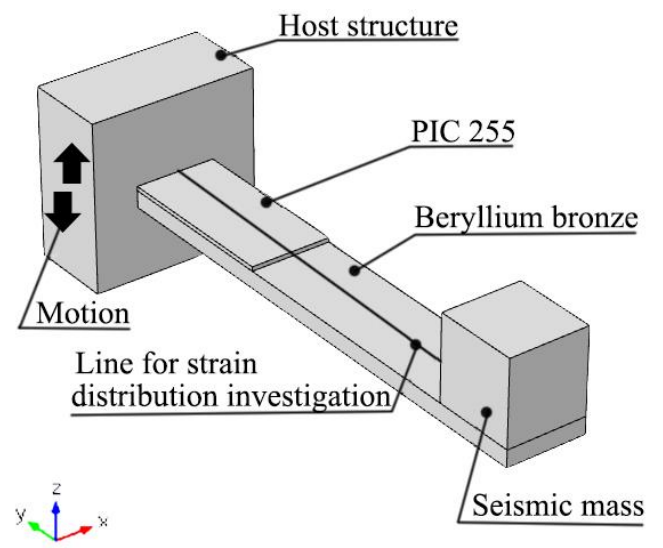

Fig. 3 The FEM model of the cantilever beam

Acceleration of the host was set to $0.5 \mathrm{~m} / \mathrm{s}^{2}$, the gravity force was included in the models as well. Resistive loads were connected in parallel to the piezo ceramic layers. The approximate resistive load value was calculated by expression 4. [5]:

$$
R_{o p t}=\frac{t_{c}}{w l_{e} \varepsilon_{33} \omega_{n}}=\frac{1}{\omega_{n} C_{p}},
$$

here $t_{c}$ is the thickness of piezoceramic layer, $w$ is width of piezo ceramic layer, $l_{e}$ is length of piezo ceramic layer, $\varepsilon_{33}$ the dielectric constant, $\omega_{n}$ natural frequency of piezoelectric cantilever, $C_{p}$ is the capacitance of piezoceramic layer. The capacitance of the piezoceramic layer was calculated as simplified parallel plate capacitor by following equation:

$$
C_{p}=\frac{\varepsilon_{33} \varepsilon_{0} A}{t}
$$

here $\varepsilon_{33}$ is the dielectric constant, $\varepsilon_{0}$ is permittivity of free space which is equal to $8.9 \cdot 10^{-12}(\mathrm{~F} / \mathrm{m}), A$ is area of electrode and $t$ is distance between two electrodes. Values of the resistive loads are listed in Table 2.

Table 2

\begin{tabular}{|c|c|}
\hline Cantilever & Value $(\mathrm{M} \Omega)$ \\
\hline Conventional & 1.415 \\
\hline Modified by cylindrical gaps & 3.675 \\
\hline Modified by rectangular gaps & 3.042 \\
\hline Modified by trapezoidal gaps & 3.694 \\
\hline
\end{tabular}

Values of the optimal resistive loads

The optimal resistive loads were calculated in order to maximize the power transfer from the source to the load. A key factor of the optimal resistance load is match to the resistance of the source. As a result, amount of power delivered to the load is the same as the power dissipated in the source. So, when both resistances matches, average power could be expressed as follows [5]:

$$
P_{a v g}=\frac{1}{2} \frac{U^{2} R_{o p t}}{\left(R_{S}+R_{o p t}\right)^{2}},
$$

here $U$ is peak voltage; $R_{S}$ is resistance of the power source. Modal analysis of piezoelectric cantilevers was performed in order to find natural frequency of the first, out - of plane, bending mode for all cantilevers. The results of modal analysis are given in Table 3. Obtained results revealed that natural frequencies of piezoelectric cantilevers are within interval $25-600 \mathrm{~Hz}$. According to Priya et. al. and Kazmierski et. al. this frequency spectrum falls to the most common ambient vibrations frequency range.

Table 3

Natural frequencies of piezoelectric cantilevers

\begin{tabular}{|c|c|}
\hline Type of piezoelectric cantilever & Natural frequency, $\mathrm{Hz}$ \\
\hline Conventional cantilever & 205.32 \\
\hline Cantilever with cylindrical gaps & 105.07 \\
\hline Cantilever with rectangular gaps & 116.07 \\
\hline Cantilever with trapezoidal gaps & 123.16 \\
\hline
\end{tabular}

Frequency domain study of all cantilevers were performed in order to analyse strain and it distribution characteristic. Also, numerical investigations of electrical characteristics such as output voltage, output current and average output power were performed as well. Analysed frequency ranges were stated with strict respect to the results 
of modal analysis. Strain and it distribution characteristics were analysed in the centre of each cantilever as shown in
Fig. 3. The strain probe was placed on the surface of the piezo ceramic layer and cantilever in order to obtain the strain characteristics along the length of piezoelectric cantilever.

Table 4

Material properties

\begin{tabular}{|c|c|c|}
\hline Material properties & Beryllium bronze DIN 2.1247 & Piezo ceramicPIC 255 \\
\hline Density, $\mathrm{kg} / \mathrm{m}^{3}$ & 8360 & 7800 \\
\hline Young's modulus, $\mathrm{N} / \mathrm{m}^{2}$ & $10^{10}$ & - \\
\hline Poisson`s ratio & 0.34 & - \\
\hline Isotropic structural loss factor & 0.02 & 0.015 \\
\hline Relative permittivity & - & $\begin{array}{l}\text { In the polarization direction } \varepsilon_{33}{ }^{T} / \varepsilon_{0}=1200 \\
\text { Perpendicular to polarity } \varepsilon_{11} T / \varepsilon_{0}=1500\end{array}$ \\
\hline Elastic stiffness coefficient $c_{33} D, \mathrm{~N} / \mathrm{m}^{2}$ & - & $16.6 \cdot 10^{10}$ \\
\hline Dielectric loss factor $-\tan \delta \times 10^{-3}$ & - & 20 \\
\hline Coupling factor $k_{31}$ & - & 0.35 \\
\hline Piezoelectric voltage coefficient $g_{31} \times 10^{-3} \mathrm{Vm} / \mathrm{N}$ & - & -11.3 \\
\hline
\end{tabular}

The subject of evaluation was strain tensor component $\varepsilon_{Y Y}$. The $\varepsilon_{Y Y}$ strain tensor is critical for electrical characteristics of the cantilevers which operates at bending modes. Results of the numerical investigations are given in Fig. 4.

Analysis of the Fig. 4 showed that strain distribution of the conventional cantilever has linear behaviour and constantly decreasing from the fixed to free end (Fig. 4, a). It can be noticed, that graph has rupture at $30 \mathrm{~mm}$. It was caused by sudden change in the thickness of piezoelectric cantilever. Additional shear deformations occur due to differences in the thickness at that point. The highest strain value of the conventional piezoelectric cantilever was obtained at frequency $205 \mathrm{~Hz}$. Maximum strain $\varepsilon_{Y Y}$ value was equal to $1.5 \times 10^{-3}$. The difference between strain value at the fixed and free end of the piezoelectric cantilever is $33.41 \%$. Hence, it can be concluded that strain of piezo ceramic is distributed unevenly and piezo ceramic layer is employed inefficiently.

Analysis of the Fig. 4, b showed that cantilever with cylindrical gaps has non - linear strain characteristic. Also, it can be noticed that strain characteristic has periodic peaks. Such behaviour of the strain characteristic was caused by periodically changes of the second moment of inertia. Validation for this assumptions can be found in equation 1 and 2. Equation 1 shows that thickness of the cantilever is dominant value which determinates value of the second moment of inertia as well as distribution function of it. Therefore, value and distribution function of second moment of inertia have direct influence to strain and it distribution. So, a function of reduced second moment of inertia was achieved by the periodic cylindrical gaps and as a result the highest strain value was obtained at $105 \mathrm{~Hz}$. Maximum strain $\varepsilon_{Y Y}$ value was equal to $9 \times 10^{-3}$. The difference between strain value at the fixed and free end of the cantilever is $22.25 \%$. The results show that maximum strain value was increased 6 times and strain distribution more than $12 \%$ compare to the conventional piezoelectric cantilever.

Graph Fig. 4, c shows that strain and it distribution characteristic at the cantilever with rectangular gaps. The strain characteristic has non - liner behaviour. Periodic strain peaks were caused by modifications made to the cross section area. The highest strain value was obtained at $116 \mathrm{~Hz}$. The maximum strain value was equal to $1.4 \times 10^{-3}$. The difference between strain values at the fixed and free end of the piezoelectric cantilever is $28.57 \%$. It can be noticed that the highest strain value is slightly lower compare to the conventional piezoelectric cantilever. On the other hand, strain distribution has better characteristic compare to the conventional piezoelectric cantilever. It was caused by geometry of the gaps i.e. at the corners of the gaps additional shear deformations were inducted and by this way strain $\varepsilon_{Y Y}$ distribution was improved.

According to this can be concluded that strain distribution was improved more than $10 \%$. Such characteristic will ensure improved usage of piezo ceramic layer compare to the conventional piezoelectric cantilever.

Results of the numerical investigation given in Fig. 4, d represents strain and strain distribution characteristic at the piezoelectric cantilever with trapezoidal gaps. Non - liner behaviour of the strain characteristic with periodic strain peaks can be observed in this case as well. The highest strain value occurred at frequency $123 \mathrm{~Hz}$. The maximum strain value was equal to $1.75 \times 10^{-3}$. The difference between strain values at the fixed and free end of the cantilever is $20.87 \%$. It can be noticed that the highest strain value obtained in this case is slightly higher compare to the conventional piezoelectric cantilever. Moreover, strain distribution was improved more than $12 \%$ compare to the conventional piezoelectric cantilever.

In order to indicate piezoelectric cantilever with the best strain characteristics the comparison of average $\varepsilon_{Y Y}$ strain was performed. Results of the comparison are given in Fig. 5.

According to the results given in Fig. 5 become obvious that the best strain characteristic has piezoelectric cantilever with the cylindrical gaps. An average strain at the piezoelectric cantilever with cylindrical gaps is 4.8 times higher compare to the conventional piezoelectric cantilever. On basis of these results can be concluded that cylindrical gaps have noticeable influence to the strain characteristics. It was caused by geometrical function of the cylindrical gap. Such geometrical function gives possibility to reduce second moment of inertia at greater cross section area.

The numerical investigations of electrical characteristics were performed. The optimal resistance loads (Table 2) were connected in parallel with piezo ceramic layer. The results of numerical investigations are given in Fig. 6. 


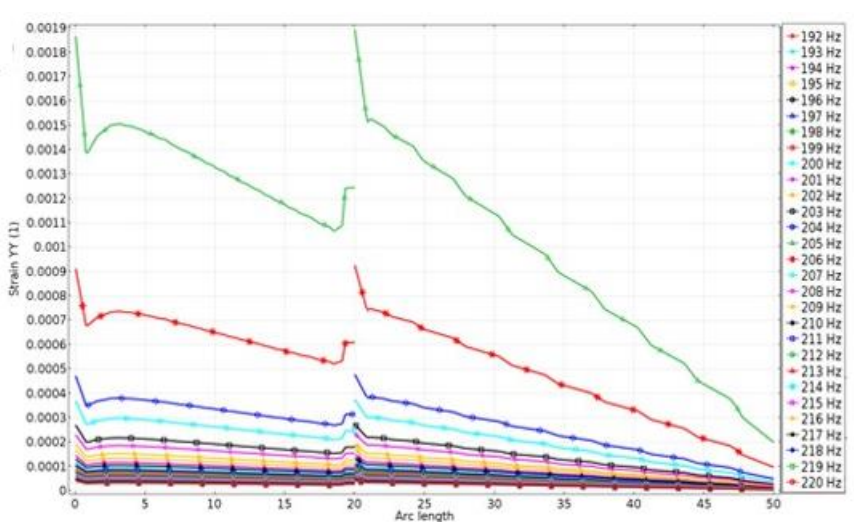

a

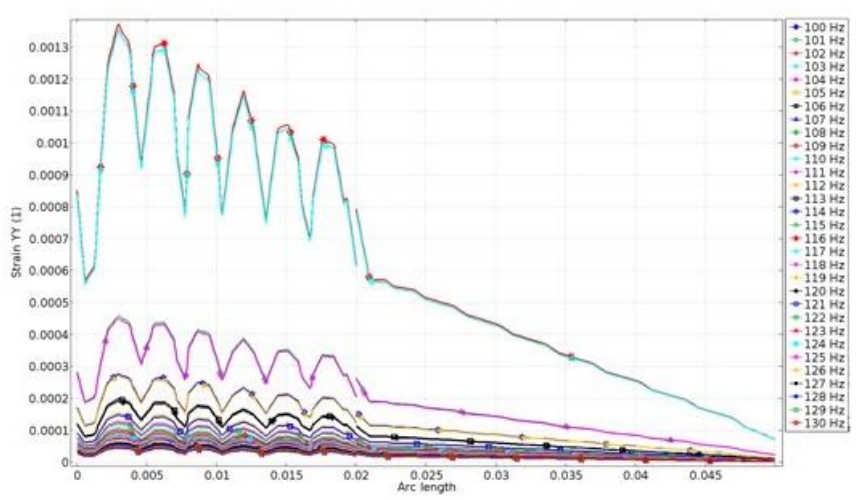

c

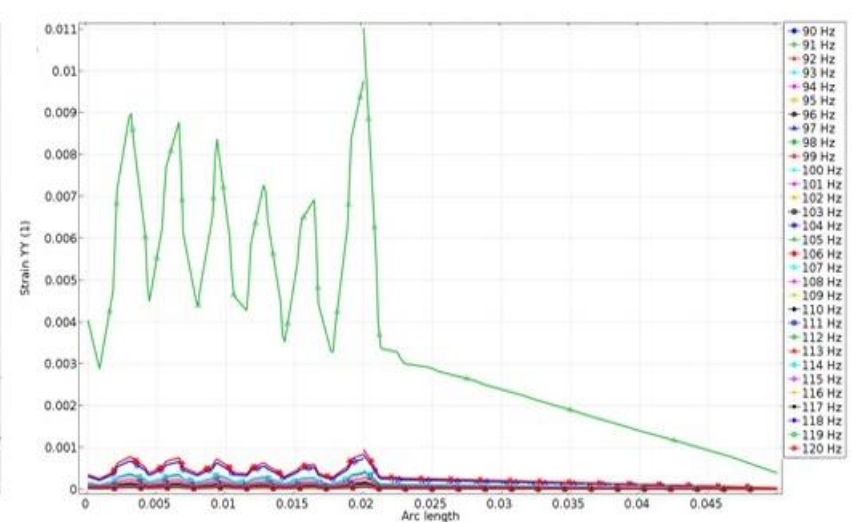

b

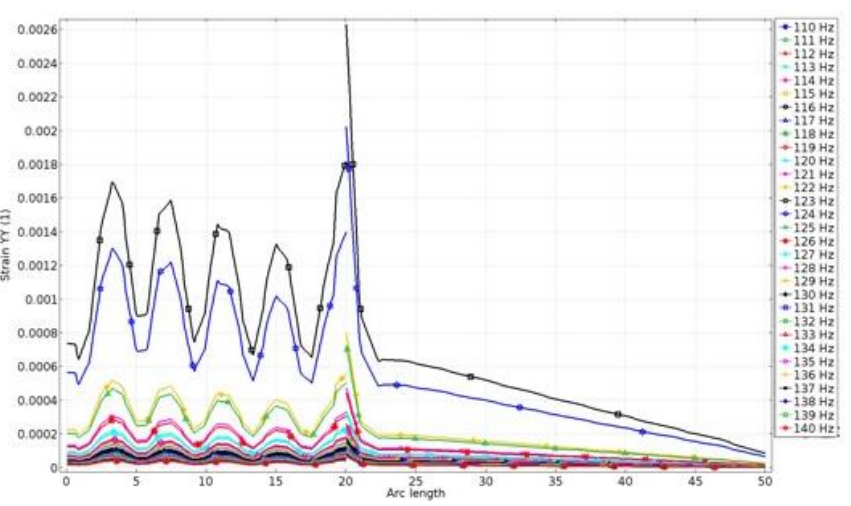

d

Fig. 4 Strain characteristics of the piezoelectric cantilevers; $a$ - conventional cantilever; $b$ - cantilever with rectangular gaps; $\mathrm{c}$ - cantilever with rectangular gaps; $\mathrm{d}$ - cantilever with trapezoidal gaps

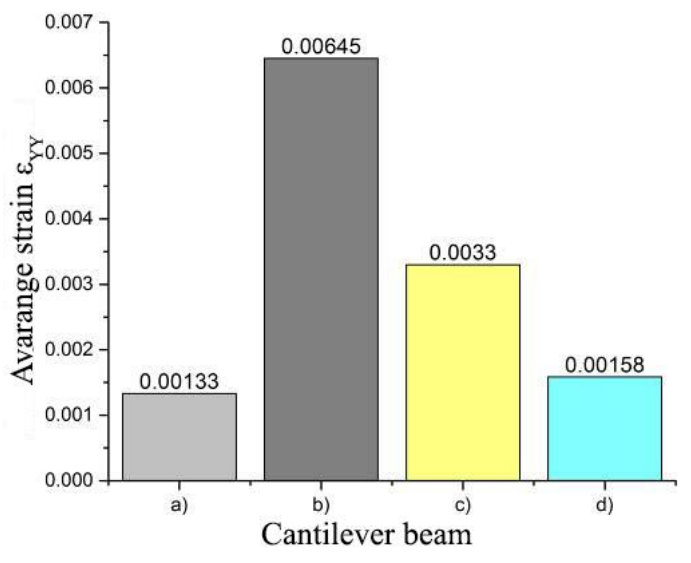

Fig. 5 Comparison of the average strain $\varepsilon_{Y Y} ;$ a - conventional cantilever, $\mathrm{b}$ - cantilever with cylindrical gaps; $\mathrm{c}-$ cantilever with rectangular gaps, $\mathrm{d}-$ cantilever with trapezoidal gaps

The electrical characteristics of conventional piezoelectric cantilever are given in Fig. 6, a. Analysis of the graphs revealed that the highest values of the electrical characteristics were obtained when a cantilevers base excitation frequency was $205 \mathrm{~Hz}$. Therefore, output voltage on the resistive load reached $15.5 \mathrm{~V}$, current at the resistance load was equal to $16.1 \mu \mathrm{A}$. An average power was equal to $0.22 \mathrm{~mW}$. Obtained values will be used as a reference data for further comparison of the piezoelectric cantilevers.

The electrical characteristics of the piezoelectric cantilever with cylindrical gaps are given in Fig.6, b. The graph revealed that electrical characteristics are at the highest level while excitation frequency equal to $105 \mathrm{~Hz}$.

The output voltage at this excitation frequency was $28.9 \mathrm{~V}$, current at the resistance load equal to $31 \mu \mathrm{A}$ and as a result average power was $0.95 \mathrm{~mW}$.

It can be noticed that output voltage is higher 1,89 times compare to the conventional piezoelectric cantilever. Output current has higher value more than 1.92 times and as a result average power has higher value more than 4.31 times. On basis of these results can be concluded that modifications of the cross section area positively affected electrical characteristics of the modified piezoelectric cantilever.

Electrical characteristics of the piezoelectric cantilever with rectangular gaps are given in Fig.6, c. It can be observed that the highest values are obtained at frequency equal to $116 \mathrm{~Hz}$. Matched operation and base excitation frequencies ensured that output voltage reached $18.2 \mathrm{~V}$, current $17.8 \mu \mathrm{A}$ and average power equal to $0.32 \mathrm{~mW}$. Compare to the conventional piezoelectric cantilever output voltage has 1.17 times higher value, current and average power are 1.10 and 1.45 times higher, respectively. The analysis of results showed that the rectangular gaps have positive influence to the electrical characteristics of the piezoelectric cantilever. However, influence is much lower compare to the case with cylindrical gaps.

The electrical characteristics of the piezoelectric cantilever with trapezoidal gaps are given in Fig. 6, d. The peak electrical characteristics were obtained at excitation frequency equal to $124 \mathrm{~Hz}$. At this excitation frequency the output voltage, current and average power reached values $19.6 \mathrm{~V}, 21 \mu \mathrm{A}$ and $0.41 \mathrm{~mW}$ respectively. Compare to the 
characteristics of the conventional piezoelectric cantilever output voltage was 1.26 times higher, current was 1.3 times higher and average power was 1.86 times higher. As in case with the rectangular gaps trapezoidal gaps have positive influence to the electrical characteristics. However, influence is much lower compare to the case with cylindrical gaps.

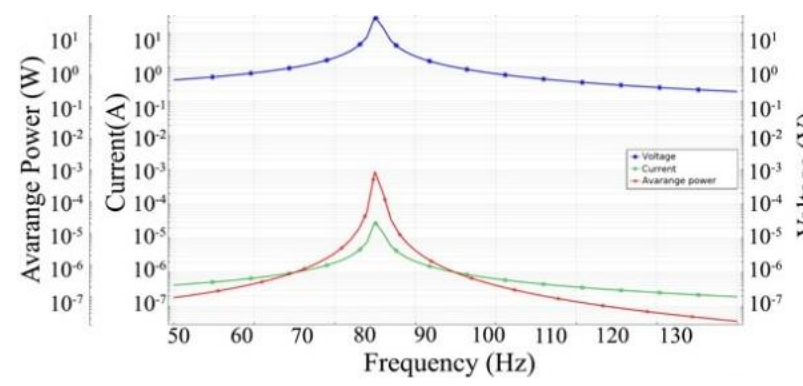

a

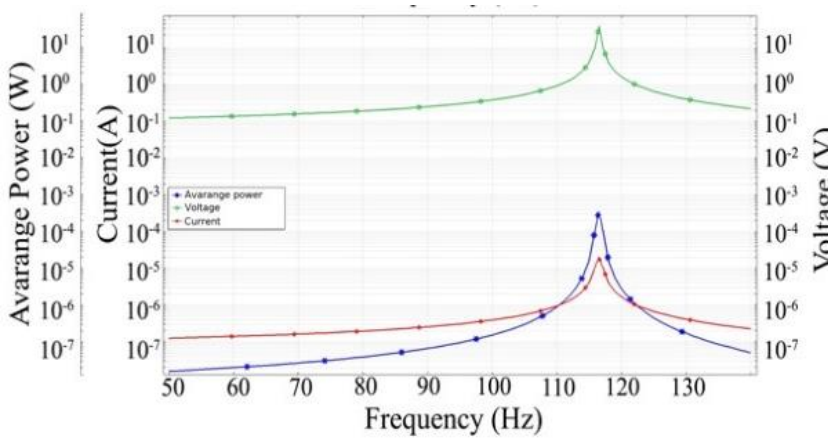

$\mathrm{c}$

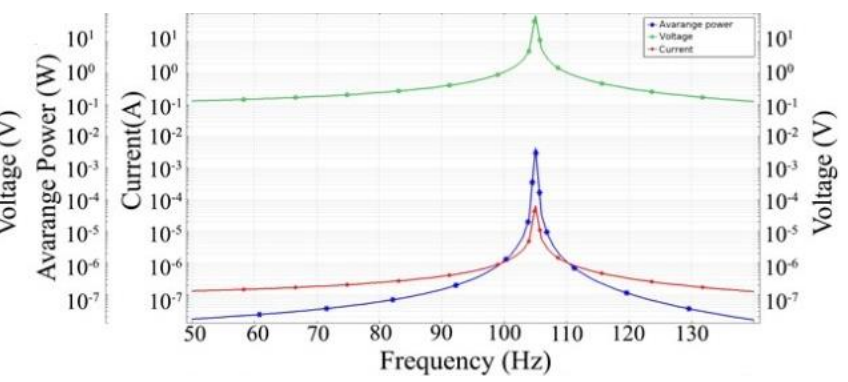

b

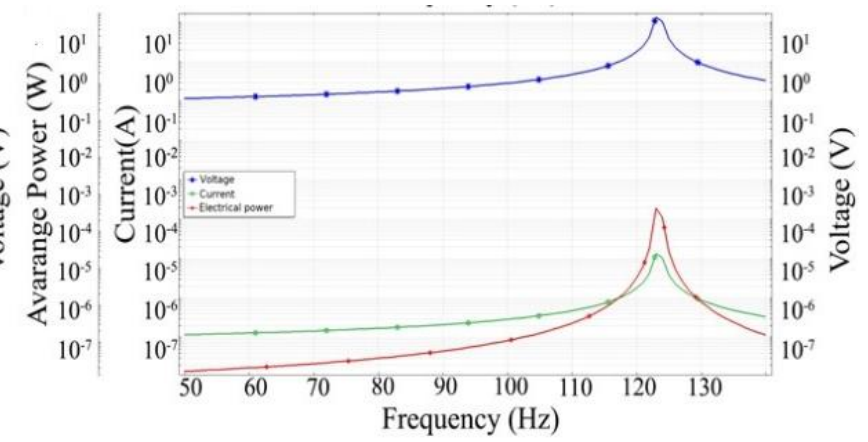

d

Fig. 6 Electrical characteristics of the piezoelectric cantilevers; a - conventional cantilever; b - cantilever with cylindrical gaps; $\mathrm{c}$ - cantilever with rectangular gaps; $\mathrm{d}$ - cantilever with trapezoidal gaps

In order to compare the electrical characteristics of piezoelectric cantilevers with different resonant frequencies derivative values must be used. Therefore, voltage, current and average power densities were used for the final comparison. The results of comparison are given in Fig. 7.

The comparison of voltage density showed that the best influence had cylindrical gaps. The piezoelectric cantilever with cylindrical gaps has $46.37 \%$ higher voltage density compare to the conventional piezoelectric cantilever and approximately $32.18 \%$ higher compare to the piezoelectric cantilevers with rectangular and trapezoidal gaps. Moreover, analysis of current density revealed that piezoelectric cantilever with cylindrical gaps has supreme value ant it is higher $48.86 \%$ compare to the conventional piezoelectric cantilever. Therefore, piezoelectric cantilevers with rectangular and trapezoidal gaps has $32.25 \%$ lower current density compare to the piezoelectric cantilever with cylindrical gaps. Finally, comparison of average power densities confirmed results analysed before and showed that cylindrical gaps have the most noticeable influence to the average power. Therefore, compare to the conventional piezoelectric cantilever, cantilever modified by cylindrical gaps has $76.91 \%$ higher average power density. Herewith, piezoelectric cantilevers, with rectangular and trapezoidal gaps have $56.82 \%$ lower average power density compare to the piezoelectric cantilever with cylindrical gaps.

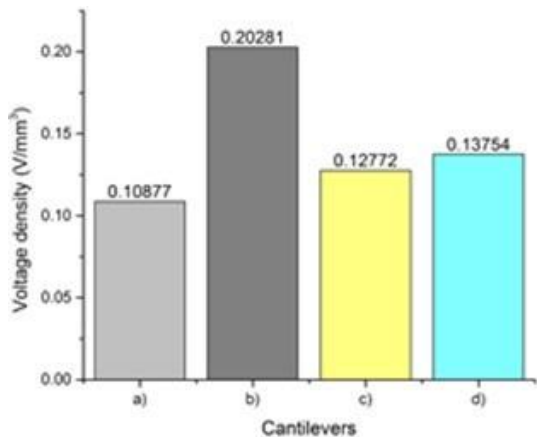

1

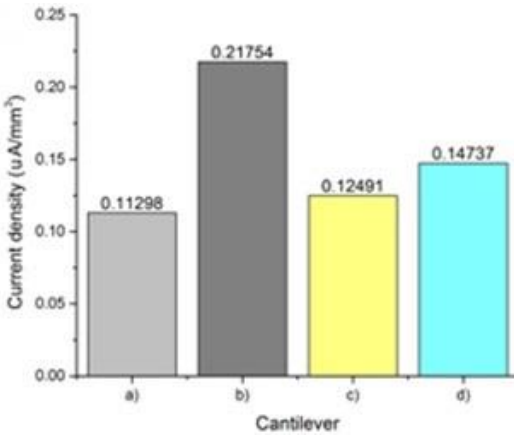

2

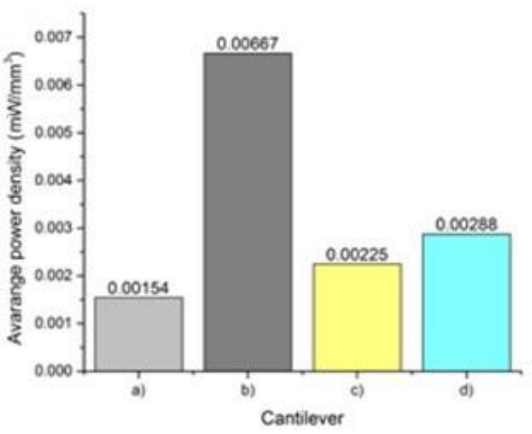

3

Fig. 7 The comparison of the electrical characteristics; 1 - voltage density; 2 - current density; 3 - average power density; a - conventional cantilever; b - cantilever with cylindrical gaps; $\mathrm{c}$ - cantilever with rectangular gaps; $d$ - cantilever with trapezoidal gaps

Summarizing numerical investigation, it can be concluded that modifications of the cross section area improved electrical characteristics of the cantilevers. The most noticeable influence to the electrical output characteristics of the cantilever beam made modifications of cross section 
area by cylindrical gaps. In order to confirm these results the experimental investigation was carried out.

\section{Experimental investigation}

The experimental investigations of electrical and mechanical characteristics were conducted to in order to confirm the results of numerical investigations. Prototypes of the piezoelectric cantilevers were made with respect to the geometrical and physical parameters used in the FEM model (Fig. 8). Firstly, operational frequencies and vibration modes of the prototypes were measured. The measurements were made by 3D vibrometer POLYTEC PSV 500. The goal of this investigation was to confirm the results of the modal analysis and experimentally indicate the first out - of plane bending mode for each piezoelectric cantilever. The results are given in Figs. 9 and 10.
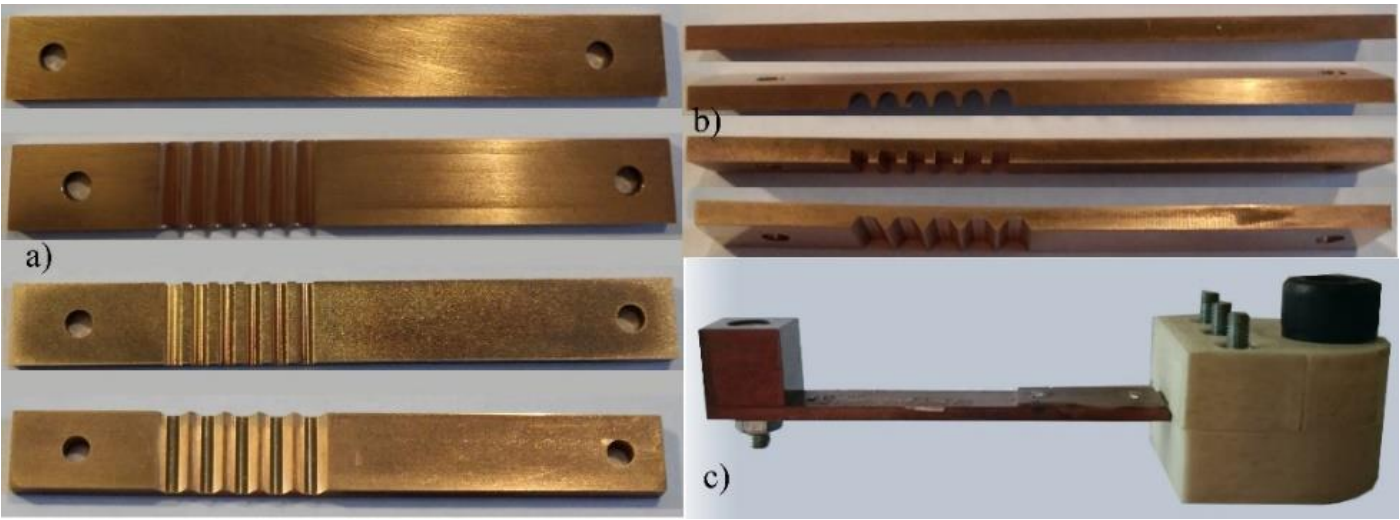

Fig. 8 Prototypes of the cantilevers; $\mathrm{a}$ - front view; $\mathrm{b}$ - side view; $\mathrm{c}$ - view of the assembled system

Fig. 9 shows vibration modes of the piezoelectric cantilevers at certain frequency. It revealed that all cantilevers operate at the first out - of plane bending mode. These results confirm results of the modal analysis conducted during numerical investigation. Comparison of operational vibration frequencies (Fig. 9) revealed that frequencies obtained numerically are slightly lower compare to the frequencies obtained by experimental investigation. The highest difference is $6.73 \%$. The differences are mainly caused by tolerances and manufacturing errors. The mismatch between frequencies is acceptable. Based on this it can be concluded that the prototypes are suitable for further investigation.

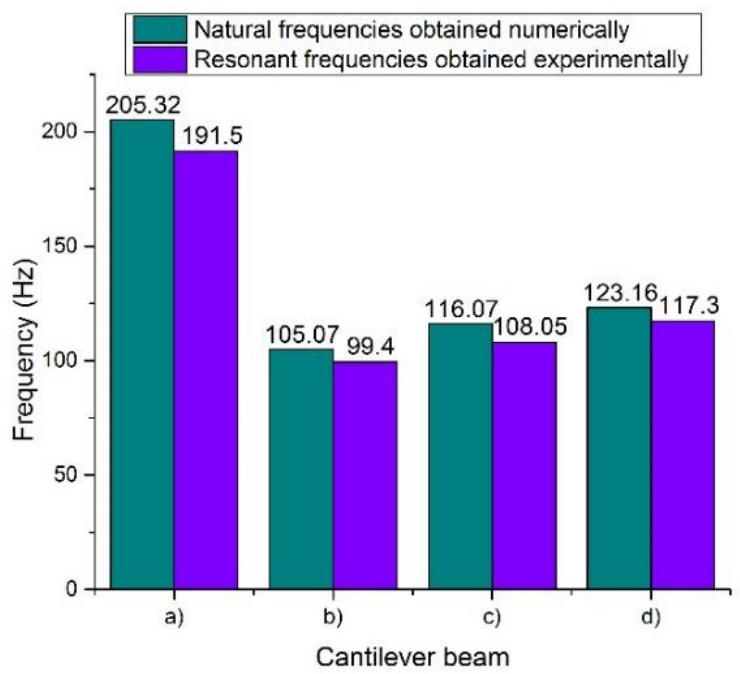

Fig. 9 Comparison of operational vibration frequencies; a conventional cantilever; $b$ - cantilever with cylindrical gaps; c - cantilever with rectangular gaps; d cantilever with trapezoidal gaps

The next step of experimental investigation was to analyse piezoelectric cantilevers response to the different excitations amplitudes and to measure electrical outputs versus resistance load. Experimental setup was built for this purpose. The principle scheme of the setup is given in Fig. 11. It consists of two optical displacement sensors used for displacement control at the base and tip of the cantilever; a special plastic clamping frame was used to attach a cantilever to an electromagnetic shaker; a computer was used for displacement data recording and analysis; a function generator and a power amplifier were employed for driving an electromagnetic shaker; a variable resistive load was used as electrical load of the cantilever; a micro current probe was used for low current measurements; an oscilloscope was used for control and recording of current and voltage values.

Measured excitation response characteristics of the piezoelectric cantilevers shows the influence of base displacement to tip displacement for each cantilever (Fig. 12, a). Displacements of the base and tip were measured by optical sensors (Fig. 11). The resistance load during this stage of investigation was equal to input resistance of the oscilloscope; i.e., $10 \mathrm{M} \Omega$. Hence, the displacement characteristics were measured in open circuit conditions. The excitation frequencies were set to the operation vibration frequencies obtained during first stage of the experimental investigation. The characteristics of the open circuit voltage versus tip displacement were investigated at same conditions. The results are given in Fig. 12, b.

In Fig. 12 - can observed that the supreme base tip displacement characteristics were achieved with the piezoelectric cantilevers modified by rectangular and trapezoidal gaps. Therefore, can be concluded that piezoelectric cantilevers with rectangular and trapezoidal gaps can response to much lower base displacement amplitudes compare to the conventional piezoelectric cantilever.

So, could be highlighted that piezoelectric cantilevers with irregular cross section area compare to the conventional piezoelectric cantilevers. Fig. 12, b shows that the highest open circuit voltage value is $22.4 \mathrm{~V}$. It was generated by the piezoelectric cantilever with cylindrical gaps. The conventional piezoelectric cantilever generated voltage 
was $10.04 \mathrm{~V}$ i.e. more than 2 times lower compare to the piezoelectric cantilever with cylindrical gaps. Such differ- ence caused by improved strain and it distribution in the piezo ceramic layer.

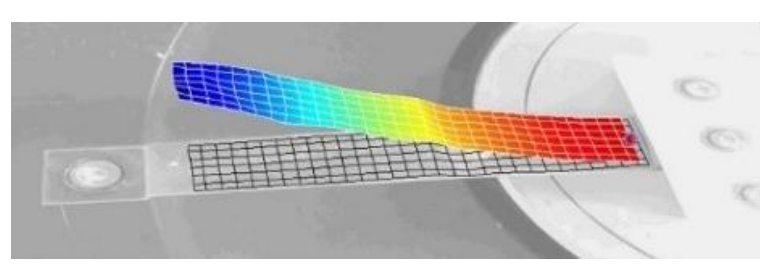

a

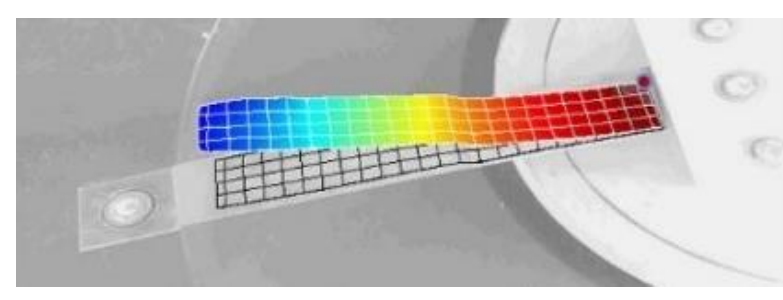

c

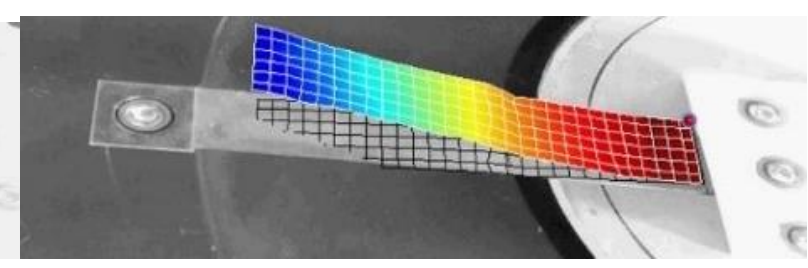

b

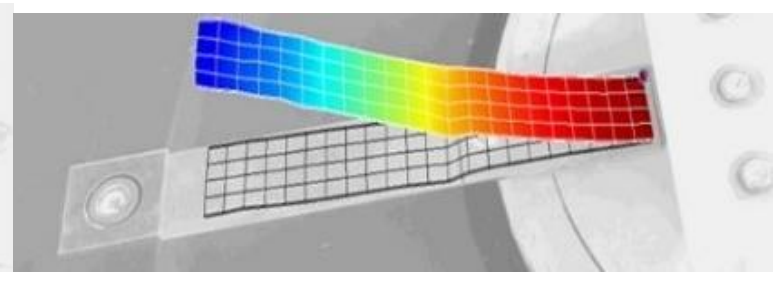

d

Fig. 10 Vibration modes of the piezoelectric cantilevers; a - conventional cantilever, $\underline{\omega}_{a}-191.5 \mathrm{~Hz}$; b cantilever with cylindrical gaps, $\omega_{b}-99.4 \mathrm{~Hz} ; \mathrm{c}-$ cantilever with rectangular gaps, $\omega_{c}-108.05 \mathrm{~Hz} ; \mathrm{d}$ - cantilever with trapezoidal gaps, $\omega_{d}-117.3 \mathrm{~Hz}$

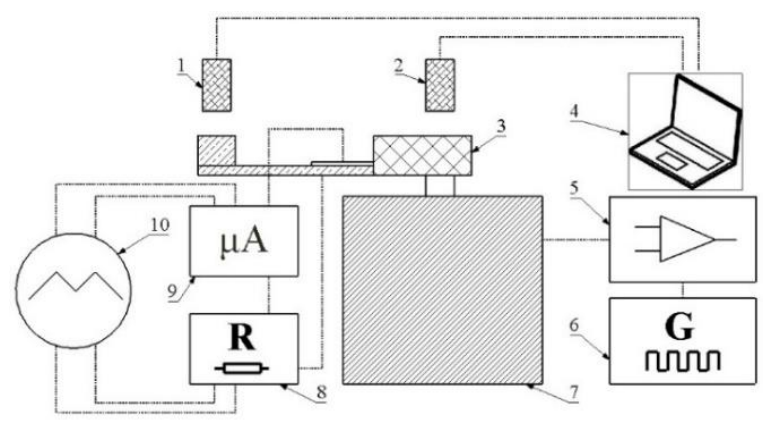

Fig. 11 Experimental setup; 1,2 - optical displacement sensor; 3 - plastic clamping frame; 4 - computer; 5 power amplifier; 6 - function generator; 7 - electromagnetic shaker; 8 - variable resistive load; 9 - micro current probe; 10 - oscilloscope

The output voltage - tip displacement characteristics were measured for each piezoelectric cantilever as well. Voltage was measured by oscilloscope as shown in Fig. 11. The variable resistance load was attached in parallel to piezo

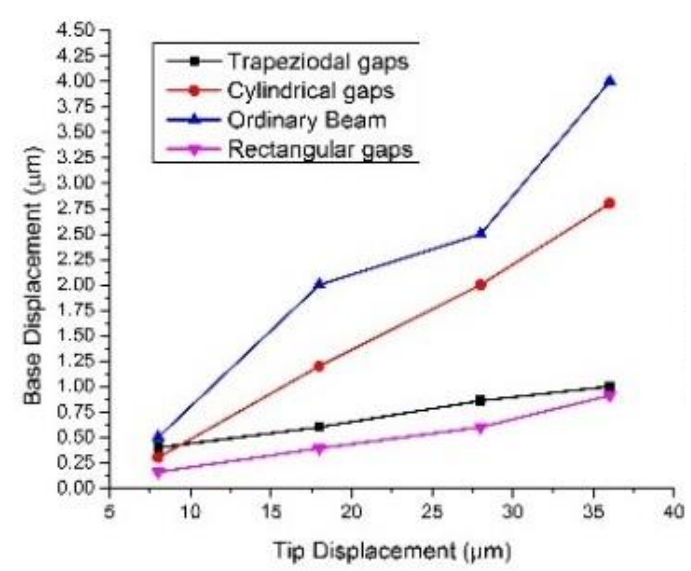

a ceramic layer. Tip displacement was controlled by the optical sensors. Results of the investigation are given in Fig. 13. As can be found in Fig. 13, the piezoelectric cantilevers with rectangular and trapezoidal gaps have noticeably higher output voltage compare to the conventional piezoelectric cantilever. The output voltage generated at $38 \mu \mathrm{m}$ tip displacement with $1 \mathrm{M} \Omega$ load was chosen as critical parameter for the comparison of cantilevers. So, output voltage $17.8 \mathrm{~V}, 18.08 \mathrm{~V}, 7.56 \mathrm{~V}$ was generated by the piezoelectric cantilevers with rectangular, trapezoidal and conventional, respectively. Therefore, the conventional piezoelectric cantilever has approximately $55 \%$ lower output voltage compare to the piezoelectric cantilevers with rectangular and trapezoidal gaps. On the other hand, the highest output voltage was generated by the piezoelectric cantilever with cylindrical gaps. The output voltage was equal to $19.1 \mathrm{~V}$. Generated voltage is higher $5.3 \%$ compare to the piezoelectric cantilevers with rectangular and trapezoidal gaps and $61 \%$ higher compare to the conventional piezoelectric cantilever. Hence, can be concluded that modifications of the cross section area by cylindrical gaps ensures $61 \%$ higher output voltage compare to the conventional cross section design.

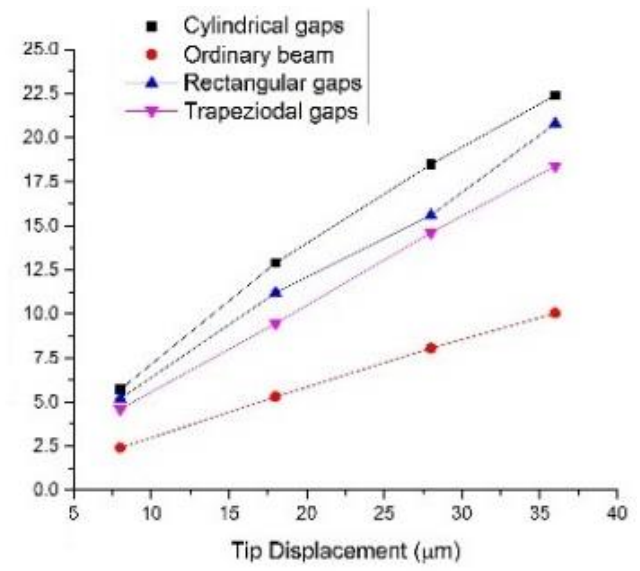

b

Fig. 12 Piezoelectric cantilevers response and open circuit voltage characteristics; a -displacement response characteristics; $\mathrm{b}$ - open circuit voltage characteristics 


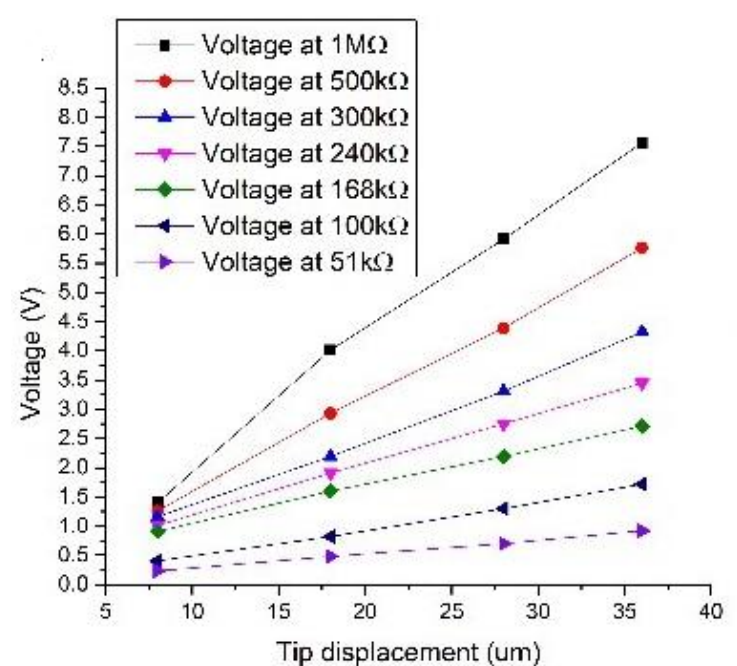

a

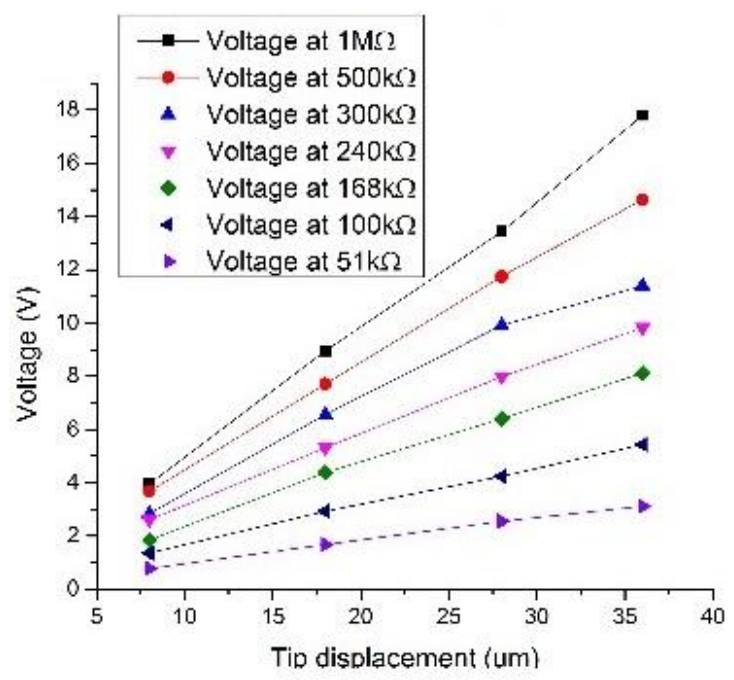

$\mathrm{c}$

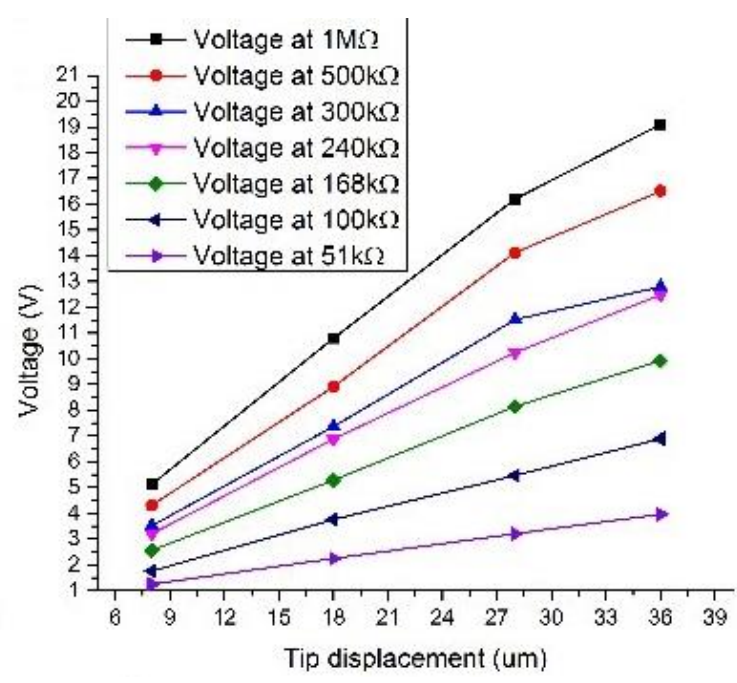

b

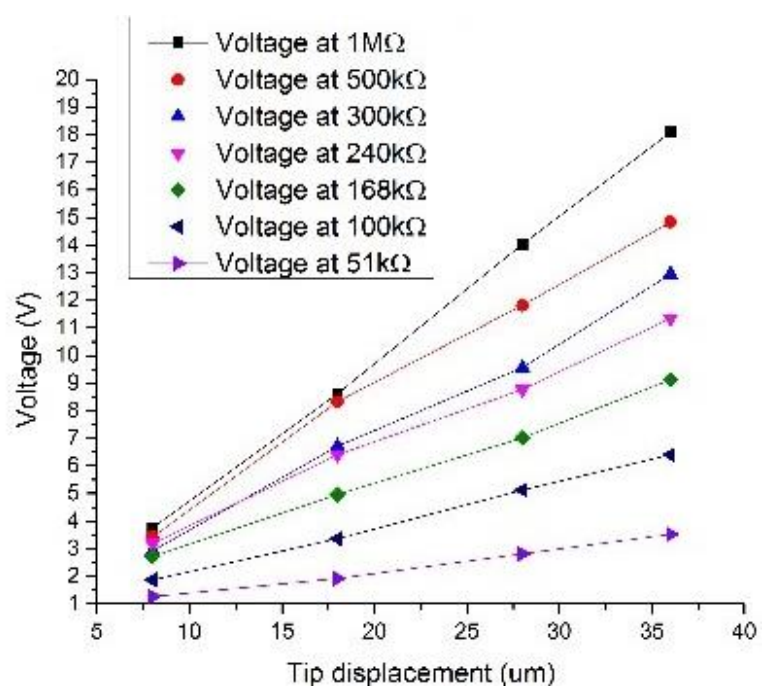

d

Fig. 13 Output voltage versus tip displacement characteristics; a - conventional cantilever; b - cantilever with cylindrical gaps; c - cantilever with rectangular gaps; $\mathrm{d}$ - cantilever with trapezoidal gaps

The output current - tip displacement characteristics were measured for each cantilever as well. As in previous case same experimental setup was used. Tip displacement was controlled by the displacement sensors, current was measured by the micro current probe. Results of the investigation are given in Fig. 14.

Fig. 14 shows that piezoelectric cantilevers with rectangular and trapezoidal gaps have much higher output current compare to the conventional piezoelectric cantilever. The output current generated at $38 \mu \mathrm{m}$ tip displacement with $51 \mathrm{k} \Omega$ load was chosen as critical parameter for the comparison of the piezoelectric cantilevers. The output current for the piezoelectric cantilevers with rectangular and trapezoidal gaps and current generated by the conventional piezoelectric cantilever are $54.7 \mu \mathrm{A}, 66.3 \mu \mathrm{A}$ and $18.3 \mu \mathrm{A}$, respectively. So, according to these results the conventional piezoelectric cantilever has approximately $72.3 \%$ lower output current compare to the cantilevers with rectangular and trapezoidal gaps. On the other hand, according to this comparison the piezoelectric cantilever with cylindrical gaps has much higher output current.
The piezoelectric cantilever with cylindrical gaps supreme over other cantilevers with $74.1 \mu \mathrm{A}$. This piezoelectric cantilever has $10.5 \%$ higher output current compare to the piezoelectric cantilevers with rectangular and trapezoidal gaps. The conventional piezoelectric cantilever has 75.3\% lower output current compare to the cantilever with cylindrical gaps. Therefore, on basis on this comparison can be concluded that modifications of the cross section area by cylindrical gaps ensured $75.3 \%$ higher output current compare to the conventional cross section design. In order to conduct accurate comparison of the piezoelectric cantilevers with different resonant frequencies derivative values were used. Therefore, output voltage, current and power densities were compared. The comparison was made with following conditions $1 \mathrm{M} \Omega$ resistance load and $38 \mu \mathrm{m}$ tip displacement. Results of the comparison are given in Fig. 15.

As can be noticed in Fig. 15 - 1 the supreme voltage density characteristic has piezoelectric cantilever with cylindrical gaps. It has approximately $5.3 \%$ higher voltage density compare to the piezoelectric cantilevers with rectangular and trapezoidal gaps and $61 \%$ higher compare to the conventional piezoelectric cantilever. Analysis of the Fig. $15-2$ revealed that cantilever with cylindrical gaps has 
$31.8 \%$ higher current density compare to the piezoelectric cantilevers with rectangular and trapezoidal gaps and $76.58 \%$ higher current density compare to the conventional piezoelectric cantilever at same conditions. In Fig. $15-3$ can be found that piezoelectric cantilever with cylindrical gaps has approximately $35.49 \%$ higher power density compare to the piezoelectric cantilevers with rectangular and trapezoidal gaps. Moreover, it has $90 \%$ higher power density compare to the conventional piezoelectric cantilever.

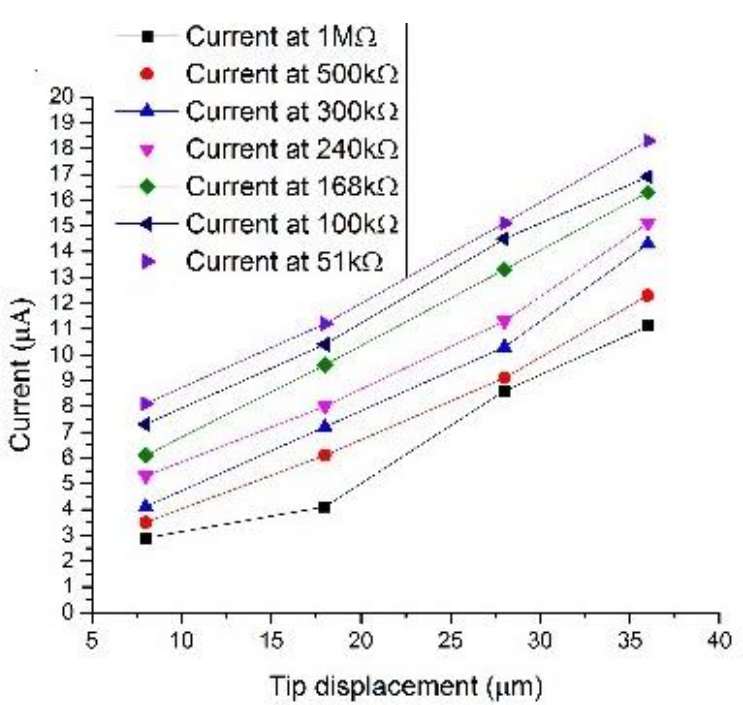

a

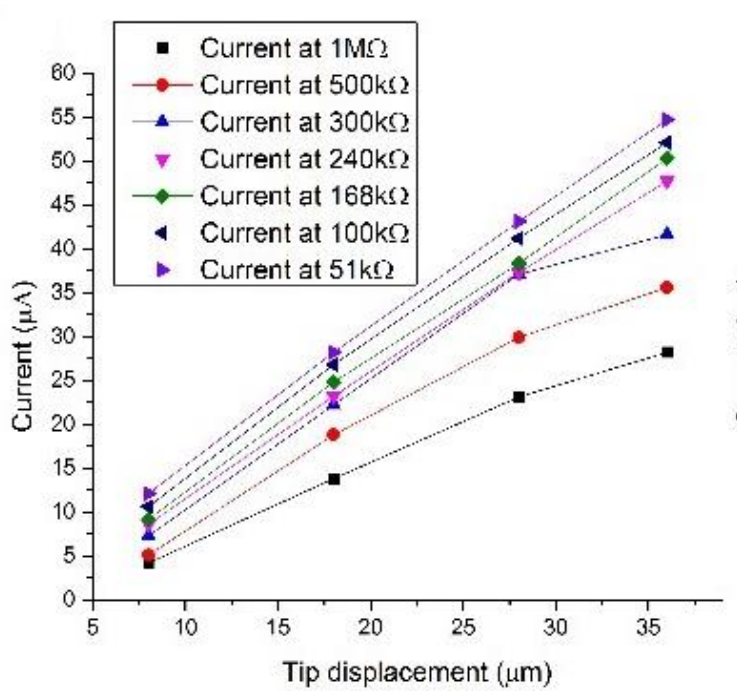

c

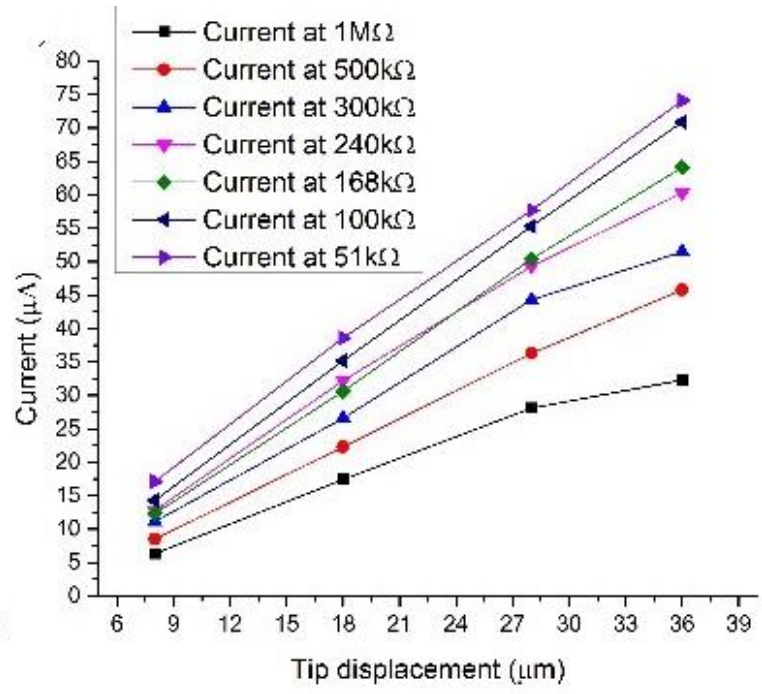

b

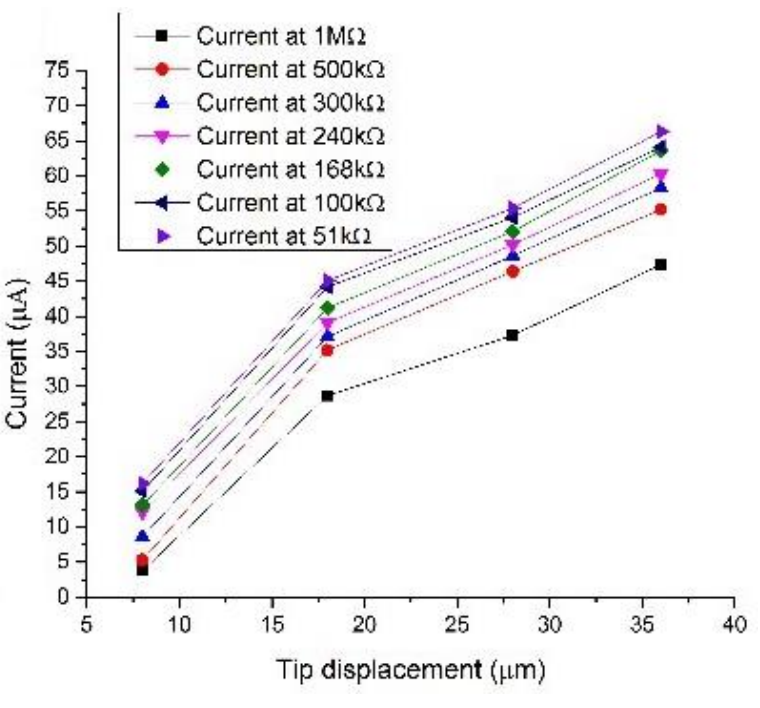

d

Fig. 14 Output current versus tip displacement characteristics; a - conventional cantilever; b - cantilever with cylindrical gaps; $\mathrm{c}$ - cantilever with rectangular gaps; $\mathrm{d}$ - cantilever with trapezoidal gaps

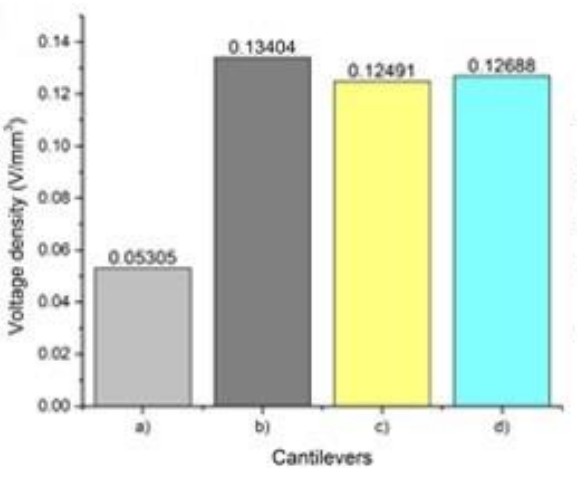

1

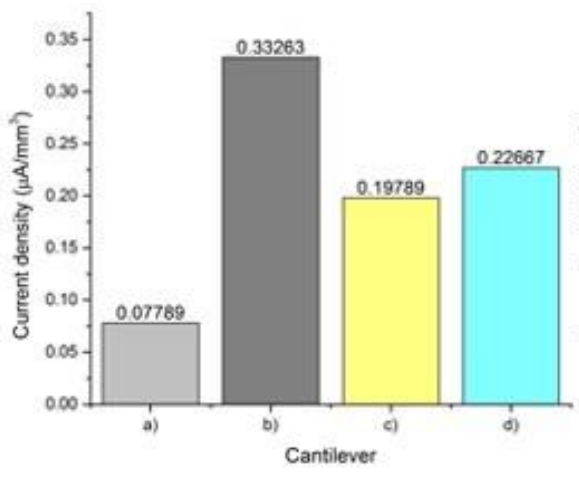

2

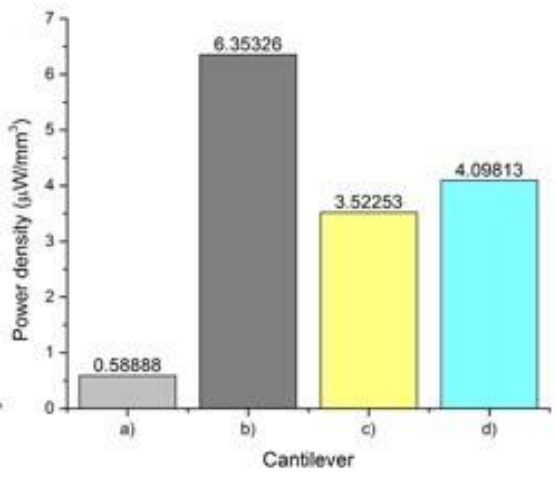

3

Fig. 15 Comparison of the electrical characteristics; 1 - voltage density; 2 - current density; 3 -power density; a - conventional cantilever; $\mathrm{b}$ - cantilever with cylindrical gaps; $\mathrm{c}$ - cantilever with rectangular gaps; $\mathrm{d}$ - cantilever with trapezoidal gaps 


\section{Conclusions}

Numerical and experimental investigations of the piezoelectric rectangular cantilevers with irregular cross section areas were performed. Numerical investigation revealed that strain and electrical characteristics of the piezoelectric cantilevers depends on the design of cross section area, cantilever thickness function and second moment of inertia. Hence, strain variation along the length of the piezoelectric cantilevers becomes non-linear because of irregular cross section area.

The experimental investigation validated results of the numerical investigation and showed that irregular cross section area design ensured noticeably improvement of the electrical characteristics of the modified piezoelectric cantilevers. The comparison of power density showed that the periodical rectangular and trapezoidal gaps used for modifications of the cross section area improved power density more than $35.49 \%$ compare to conventional piezoelectric cantilever. Moreover, cylindrical gaps improved power density more than $90 \%$ at same conditions.

\section{References}

1. Erturk, A.; Inman, D.J. 2011. Piezoelectric energy harvesting, John Wiley and Sons. https://doi.org/10.1002/9781119991151.

2. Roundy,S.; Kenneth, P.; Wright, Rabaey, J.M. 2004. Energy Scavenging for Wireless Sensor Networks, Springer Science + Business Media, New York. http://dx.doi.org/10.1007/978-1-4615-0485-6.

3. Priya, S.; Inman, D.J. 2009. Energy Harvesting Technologies. Springer Science + Business Media, New York. http://dx.doi.org/10.1007/978-0-387-76464-1.

4. Spreemann, D.; Manoli, Y. 2012. Electromagnetic Vibration Energy Harvesting Devices. Springer Science + Business Media, New York. http://dx.doi.org/10.1007/978-94-007-2944-5.

5. Kazmierski, T.J.; Beeby, S. 2011. Energy Harvesting Systems. Springer Science + Business Media, New York. http://dx.doi.org/10.1007/978-1-4419-7566-9.

6. Lu, F.; Lee, H. P.; Lim, S.P. 2003. Modeling and analysis of micro piezoelectric power for wireless sensor networks. IEEE Sensors. http://dx.doi.org/10.1109/ICSENS.2009.5398559.

7. Muthalif, A. G. A.; Nordin, N. H. D. 2015. Optimal piezoelectric beam shape for single and broadband vibration energy harvesting: Modeling, simulation and experimental results. Mechanical Systems and Signal Processing 54. http://dx.doi.org/10.1016/j.ymssp.2014.07.014.

8. Paquin, S.; St-Amant, Y. 2010. Improving the performance of a piezoelectric energy harvester using a variable thickness beam. Smart Materials and Structures. http://dx.doi.org/10.1088/0964-1726/19/10/105020.
9. Reddy, A.R.; Umapathy, M.; Ezhilarasi, D.; Uma, G. 2015. Cantilever beam with trapezoidal cavity for improved energy harvesting. International Journal of Precision Engineering and Manufacturing. http://dx.doi.org/10.1007/s12541-015-0244-5.

10. Guan, Q. C.; Ju, B.; Xu, J. W.; Liu, Y. B.; Feng, Z. H. 2013. Improved strain distribution of cantilever piezoelectric energy harvesting devices using H-shaped proof masses. Journal of Intelligent Material Systems and Structures. http://dx.doi.org/10.1177/1045389X13476150.

11. Xu, J. W.; Liu, Y. B.; Shao, W. W.; Feng, Z. 2012. Optimization of a right-angle piezoelectric cantilever using auxiliary beams with different stiffness levels for vibration energy harvesting. Smart Materials and Structures. http://dx.doi.org/10.1088/0964-1726/21/6/065017.

12. Ma, X.; Wilson, A.; Rahn, C.D.; Trolier-McKinstry, S. 2016. Efficient Energy Harvesting Using Piezoelectric Compliant Mechanisms: Theory and Experiment. Journal of Vibration and Acoustics. http://dx.doi.org/10.1115/1.4032178.

\section{A. Čeponis, D. Mažeika, G. Kulvietis and Y.Ying}

\section{PIEZOELECTRIC CANTILEVERS FOR ENERGY HARVESTING WITH IRREGULAR DESIGN OF THE CROSS SECTIONS}

S u m m a r y

Results of the numerical and experimental investigations of the piezoelectric rectangular cantilevers with irregular design of the cross section area are presented in this paper. The aim of the investigation was to analyse how modification of the cross section area by periodical gaps acts on power density of the piezoelectric energy harvesting systems based on a rectangular cantilever. It was found out, that modifications of the cross section area ensure higher strain values and allows to improve strain distribution in piezo ceramic layer and by this way to improve power density of the energy harvesting systems. Numerical investigations of the piezoelectric cantilevers with irregular design of the cross section area were performed in order to analyse strain distribution and predict electrical characteristics of the improved energy harvesting systems. Experimental investigations of the prototypes were performed and results of the numerical modelling were validated. Results of the numerical and experimental investigation are discussed as well.

Keywords: piezoelectric energy harvesting, irregular cross section design, rectangular cantilever beam.

Received April 21, 2017

Accepted April 18, 2018 